\title{
Maximum principles for time-fractional Caputo-Katugampola diffusion equations
}

\author{
Liang $\mathrm{Cao}^{\mathrm{a}}$, Hua Kong ${ }^{\mathrm{b}}$, Sheng-Da Zeng ${ }^{\mathrm{b}, \mathrm{c}, *}$ \\ a School of Automation, Guangdong University of Technology, Guangzhou, 510006, P. R. China. \\ ${ }^{b}$ Data Recovery Key Laboratory of Sichuan Province, College of Mathematics and Information Science, Neijiang Normal University, \\ Neijiang 641100, P. R. China. \\ ${ }^{c}$ Institute of Computer Science, Faculty of Mathematics and Computer Science, Jagiellonian University, ul. Lojasiewicza 6, 30-348 \\ Krakow, Poland.
}

Communicated by X.-J. Yang

\begin{abstract}
Maximum and minimum principles for time-fractional Caputo-Katugampola diffusion operators are proposed in this paper. Several inequalities are proved at extreme points. Uniqueness and continuous dependence of solutions for fractional diffusion equations of initial-boundary value problems are considered. (C)2017 All rights reserved.
\end{abstract}

Keywords: Caputo-Katugampola fractional operators, fractional diffusion equations, maximum principles, uniqueness, continuous dependence.

2010 MSC: 49J53, 49K40, 90C33, 90C46.

\section{Introduction}

Fractional order integral and differential equations have been demonstrated to be an efficient tool in numerous applications, for example, fractal phenomena [3, 23-28], contact mechanics problems [15], control problems [20], fluid mechanics problems [16] and so on [4, 21, 22, 30].

The maximum and minimum principles of fractional partial differential equations have captured special attention in mathematical analysis. Because they enable to obtain some important propositions of solutions for partial differential systems, such as the existence and uniqueness of solution. They were systematically introduced by Luchko in [12] in which the existence and uniqueness of solution for timefractional diffusion equations were obtained. Then the maximum principles for Riemann-Liouville type operators were established in [1]. Recently, Al-Refai et al. and Ye et al. gave the maximum principles to the multi-term Riemann-Liouville cases [2] and the multi-term Riesz-Caputo space equations [29], respectively. Liu et al. [11] proved the generalized maximum and minimum principles for multi-term spacetimes variable-order fractional diffusion equations. On the other hand, using those maximum principles

\footnotetext{
${ }^{*}$ Corresponding author

Email addresses: bdhzxcaoliang@163.com (Liang Cao), konghua2008@126.com (Hua Kong), shdzeng@hotmail.com; zengshengda@163. com (Sheng-Da Zeng)

doi:10.22436/jnsa.010.04.75
} 
mentioned above, a formal solution of a Fourier series in regard to the eigenfunctions of Sturm-Liouville eigenvalue problems were achieved in [13], and non-axisymmetric solutions to time-fractional diffusionwave equation in an infinite cylinder were established by Povstenko [18]. For more details on this topics readers are suggested to consult $[6,7,14]$ and the references therein.

Consider the following time-fractional Caputo-Katugampola (C-K) diffusion equation

$$
\mathrm{P}_{\alpha, \rho}(\mathrm{u})=\mathrm{F}(\mathrm{t}, \mathrm{x}, \mathrm{u}), \quad(\mathrm{t}, \mathrm{x}) \in \Omega_{\mathrm{T}}:=(0, \mathrm{l}) \times(0, \mathrm{~T}),
$$

with the following initial-boundary conditions

$$
\left\{\begin{array}{l}
u(0, t)=g_{1}(t), \quad t \in[0, T], \\
u(l, t)=g_{2}(t), \quad t \in[0, T]
\end{array}\right.
$$

and

$$
u(x, 0)=\Phi(x), \quad x \in[0, l] .
$$

In this paper, $\mathrm{P}_{\alpha, \rho}$ is called to be the fractional $\mathrm{C}-\mathrm{K}$ diffusion operator and defined by

$$
\mathrm{P}_{\alpha, \rho}(\mathrm{u})={ }^{\mathrm{C}} \mathrm{D}_{0^{+}}^{\alpha, \rho} \mathrm{u}(\mathrm{x}, \mathrm{t})-\mathrm{Lu},
$$

where $L$ is a second order differential operator

$$
L u=a(x, t) u_{x x}+b(x, t) u_{x}+c(x, t) u,
$$

and ${ }^{C} D_{a^{+}}^{\alpha, \rho}$ is the generalized fractional derivative (see Definition 2.10, below) with $\alpha \in(0,1)$ and $\rho>0$ defined by

$$
{ }^{C} D_{0^{+}}^{\alpha, \rho} f(t):=\frac{\rho^{\alpha} t^{1-\rho}}{\Gamma(1-\alpha)} \frac{d}{d t} \int_{0}^{t} \frac{s^{\rho-1}}{\left(t^{\rho}-s^{\rho}\right)^{\alpha}}[f(s)-f(0)] d s .
$$

Let the parameter $\rho=1$. Then we can obtain the time-fractional Caputo diffusion equation,

$$
\frac{1}{\Gamma(1-\alpha)} \int_{0}^{t}(t-s)^{-\alpha} u_{t}(s, x) d s=L u+F(t, x, u), \quad(t, x) \in \Omega_{\mathrm{T}}:=(0, l) \times(0, T),
$$

which has been studied by Luchko [12], Al-Refai and Luchko [2] and Ye et al. [29].

The rest of the paper is structured as follows. In Section 2 we review some preliminary material needed in the study of time-fractional C-K diffusion equation. The maximum and minimum principles fractional C-K diffusion operator, (1.1), were obtained in Section 3. Section 4 is devoted to prove the uniqueness and continuous dependence of solution for time-fractional $\mathrm{C}-\mathrm{K}$ diffusion equation by use of the maximum principles.

\section{Preliminaries}

In this paper we recall the basic notation and some results which are needed in the sequel, see [10, 17, 19, 20].

Definition 2.1 (Riemann-Liouville fractional integral). Let $f \in \mathrm{L}^{1}[\mathrm{a}, \mathrm{b}]$ and $\alpha>0$. The Riemann-Liouville (R-L) fractional integral of order $\alpha$ with the lower limit zero for the function $f:[a, b] \rightarrow \mathbb{R}$ can be written as

$$
I_{a^{+}}^{\alpha} f(t):=\frac{1}{\Gamma(\alpha)} \int_{a}^{t}(t-s)^{\alpha-1} f(s) d s,
$$

provided that the right side is point-wise defined on $[a, b]$, where $\Gamma$ is the well-known gamma function defined by

$$
\Gamma(\alpha):=\int_{0}^{+\infty} t^{\alpha-1} e^{-t} d t
$$


Definition 2.2 (R-L fractional derivative). Let $f \in \mathrm{L}^{1}[\mathrm{a}, \mathrm{b}]$ and $\alpha \in(0,1]$. The R-L fractional derivative of order $\alpha$ with the lower limit zero for function $f:[a, b] \rightarrow \mathbb{R}$ can be expressed as

$$
D_{a^{+}}^{\alpha} f(t):=\frac{1}{\Gamma(1-\alpha)} \frac{d}{d t} \int_{0}^{t}(t-s)^{-\alpha} f(s) d s
$$

Definition 2.3 (Caputo fractional derivative). Let $f \in \mathrm{L}^{1}[\mathrm{a}, \mathrm{b}]$ and $\alpha \in(0,1]$. The Caputo fractional derivative of order $\alpha$ for function $f:[a, b] \rightarrow \mathbb{R}$ can be expressed as

$$
{ }^{c} D_{a^{+}}^{\alpha} f(t):=\frac{1}{\Gamma(1-\alpha)} \frac{d}{d t} \int_{a}^{t}(t-s)^{-\alpha}[f(s)-f(a)] d s .
$$

Remark 2.4. Particularly, if $f$ is of class $C^{1}[a, b]$, then we have an equivalent form of (2.2)

$$
{ }^{c} D_{a^{+}}^{\alpha} f(t)=\frac{1}{\Gamma(1-\alpha)} \int_{a}^{t}(t-s)^{-\alpha} f^{\prime}(s) d s .
$$

We also recall the Caputo-Hadamard integral and derivative operators, see $[10,17,19,20]$.

Definition 2.5 (Riemann-Hadamard fractional integral). Let $f \in \mathrm{L}^{1}[\mathrm{a}, \mathrm{b}]$ and $\alpha>0$. The RiemannHadamard (R-H) fractional integral of order $\alpha$ for function $f:[a, b] \rightarrow \mathbb{R}$ can be expressed as

$$
\mathrm{H}_{\mathrm{I}_{\mathrm{a}^{+}}^{\alpha}} \mathrm{f}(\mathrm{t}):=\frac{1}{\Gamma(\alpha)} \int_{\mathrm{a}}^{\mathrm{t}} \frac{\mathrm{f}(\mathrm{s})}{\mathrm{s}(\ln \mathrm{t}-\ln s)^{1-\alpha}} \mathrm{ds} .
$$

Definition 2.6 (Caputo-Hadamard fractional derivative). Let $f \in \mathrm{L}^{1}[\mathrm{a}, \mathrm{b}]$ and $\alpha \in(0,1]$. The CaputoHadamard (C-H) fractional derivative of order $\alpha$ for function $f:[a, b] \rightarrow \mathbb{R}$ can be expressed as

$$
{ }^{H} D_{a^{+}}^{\alpha} f(t):=\frac{t}{\Gamma(1-\alpha)} \frac{d}{d t} \int_{a}^{t}(\ln t-\ln s)^{-\alpha} \frac{f(s)-f(a)}{s} d s
$$

Remark 2.7. Similarly, when $f \in C^{1}[a, b]$, then we get the equivalent form of (2.4)

$$
{ }^{H} D_{a^{+}}^{\alpha} f(t)=\frac{1}{\Gamma(1-\alpha)} \int_{a}^{t}(\ln t-\ln s)^{-\alpha} f^{\prime}(s) d s .
$$

We now introduce the generalized fractional operators (one can refer to $[8,9]$ ) as follows, which will be used in the sequel to consider the time fractional diffusion problems.

Definition 2.8 (Riemann-Katugampola fractional integral). Let $f \in \mathrm{L}^{1}[\mathrm{a}, \mathrm{b}], \alpha>0$ and $\rho>0$. The Riemann-Katugampola (R-K) fractional integral of $\alpha$ for function $f:[a, b] \rightarrow \mathbb{R}$ with respect to parameter $\rho$ can be expressed as

$$
I_{a^{+}}^{\alpha, \rho} f(t):=\frac{\rho^{1-\alpha}}{\Gamma(\alpha)} \int_{a}^{t} \frac{s^{\rho-1} f(s)}{\left(t^{\rho}-s^{\rho}\right)^{1-\alpha}} d s .
$$

Remark 2.9. It is obvious that R-K fractional integral (2.5) reduces to R-L fractional integral (2.1) for $\rho=1$. In addition, taking $\rho \downarrow 0^{+}$, we have by the L'Hospital rule

$$
\begin{aligned}
\lim _{\rho \downarrow 0^{+}} \mathrm{I}_{\mathrm{a}^{+}}^{\alpha, \rho} \mathrm{f}(\mathrm{t}) \mathrm{dt} & =\frac{1}{\Gamma(\alpha)} \int_{\mathrm{a}}^{\mathrm{t}} \lim _{\rho \downarrow 0^{+}} \mathrm{f}(\mathrm{s}) \mathrm{s}^{\rho-1}\left(\frac{\mathrm{t}^{\rho}-\mathrm{s}^{\rho}}{\rho}\right)^{\alpha-1} \mathrm{ds} \\
& =\frac{1}{\Gamma(\alpha)} \int_{a}^{t} \frac{\mathrm{f}(\mathrm{s})}{\mathrm{s}(\ln \mathrm{t}-\ln s)^{1-\alpha}} \mathrm{ds},
\end{aligned}
$$

which implies that C-K fractional integral (2.5) can be reduced to C-H fractional integral (2.3), for $\rho \downarrow 0^{+}$. 
Also, we give the following C-K fractional derivative.

Definition 2.10 (C-K fractional derivative). Let $f \in \mathrm{L}^{1}[\mathrm{a}, \mathrm{b}], \alpha \in(0,1]$ and $\rho>0$. The C-K fractional derivative of $\alpha$ for function $f:[a, b] \rightarrow \mathbb{R}$ with respect to parameter $\rho$ can be expressed as

$$
{ }^{C} D_{a^{+}}^{\alpha, \rho} f(t):=\frac{\rho^{\alpha} t^{1-\rho}}{\Gamma(1-\alpha)} \frac{d}{d t} \int_{a}^{t} \frac{s^{\rho-1}}{\left(t^{\rho}-s^{\rho}\right)^{\alpha}}[f(s)-f(a)] d s .
$$

The following lemma gives an equivalent form of $C-K$ fractional derivative (2.6), when $f \in C^{1}[a, b]$.

Lemma 2.11. Assume that $\mathrm{f}:[\mathrm{a}, \mathrm{b}] \rightarrow \mathbb{R}$ is satisfied to $\mathrm{f} \in \mathrm{C}^{1}[\mathrm{a}, \mathrm{b}]$, then the $C-K$ fractional operator (2.6) has the following equivalent form

$$
\begin{aligned}
{ }^{C} D_{a^{+}}^{\alpha, \rho} f(t) & =\frac{\rho^{\alpha}}{\Gamma(1-\alpha)} \int_{a}^{t} \frac{f^{\prime}(s)}{\left(t^{\rho}-s^{\rho}\right)^{\alpha}} d s \\
& =I_{a^{+}}^{1-\alpha, \rho}\left(s^{1-\rho} \frac{d}{d s} f\right)(t) .
\end{aligned}
$$

Proof. We easily calculate

$$
\begin{aligned}
\frac{\rho^{\alpha}}{\Gamma(1-\alpha)} t^{1-\rho} \frac{d}{d t} \int_{a}^{t} \frac{s^{\rho-1}}{\left(t^{\rho}-s^{\rho}\right)^{\alpha}} f(a) d s & =\frac{\rho^{\alpha}}{\Gamma(1-\alpha)} t^{1-\rho} \frac{d}{d t}\left(f(a) \int_{a^{\rho}}^{t^{\rho}} \frac{d s}{\rho\left(t^{\rho}-s\right)^{\alpha}} d s\right) \\
& =\frac{\rho^{\alpha}}{\Gamma(1-\alpha)} t^{1-\rho} \frac{d}{d t}\left(-\left.\frac{f(a)}{(1-\alpha) \rho}\left(t^{\rho}-s\right)^{1-\alpha}\right|_{a^{\rho}} ^{t^{\rho}}\right) \\
& =\frac{\rho^{\alpha}}{\Gamma(1-\alpha)} t^{1-\rho} \frac{d}{d t}\left(\frac{f(a)}{(1-\alpha) \rho}\left(t^{\rho}-a^{\rho}\right)^{1-\alpha}\right) .
\end{aligned}
$$

Considering the fact $f \in C^{1}[a, b]$, we take integrating by parts to obtain

$$
\begin{aligned}
\frac{\rho^{\alpha} t^{1-\rho}}{\Gamma(1-\alpha)} \frac{d}{d t} & \int_{a}^{t} \frac{s^{\rho-1}}{\left(t^{\rho}-s^{\rho}\right)^{\alpha}} f(s) d s \\
& =\frac{\rho^{\alpha} t^{1-\rho}}{\Gamma(1-\alpha)} \frac{d}{d t}\left[-\left.\frac{\left(t^{\rho}-s^{\rho}\right)^{1-\alpha}}{\rho(1-\alpha)} f(s)\right|_{a} ^{t}+\frac{1}{(1-\alpha)} \int_{a}^{t} \frac{\left(t^{\rho}-s^{\rho}\right)^{1-\alpha} f^{\prime}(s)}{\rho} d s\right] \\
& =\frac{\rho^{\alpha} t^{1-\rho}}{\Gamma(1-\alpha)} \frac{d}{d t}\left(\frac{\left(t^{\rho}-a^{\rho}\right)^{1-\alpha}}{\rho(1-\alpha)} f(a)\right)+\frac{\rho^{\alpha} t^{1-\rho}}{\rho \Gamma(2-\alpha)} \frac{d}{d t} \int_{a}^{t}\left(t^{\rho}-s^{\rho}\right)^{1-\alpha} f^{\prime}(s) d s \\
& =\frac{\rho^{\alpha} t^{1-\rho}}{\Gamma(1-\alpha)} \frac{d}{d t}\left(\frac{\left(t^{\rho}-a^{\rho}\right)^{1-\alpha}}{\rho(1-\alpha)} f(a)\right)+\frac{\rho^{\alpha}}{\Gamma(1-\alpha)} \int_{a}^{t}\left(t^{\rho}-s^{\rho}\right)^{-\alpha} f^{\prime}(s) d s .
\end{aligned}
$$

From (2.7), we directly get

$$
\begin{aligned}
{ }^{C} D_{a^{+}}^{\alpha, \rho} f(t) & =\frac{\rho^{\alpha} t^{1-\rho}}{\Gamma(1-\alpha)} \frac{d}{d t} \int_{a}^{t} \frac{s^{\rho-1}}{\left(t^{\rho}-s^{\rho}\right)^{\alpha}} f(s) d s-\frac{\rho^{\alpha} t^{1-\rho}}{\Gamma(1-\alpha)} \frac{d}{d t} \int_{a}^{t} \frac{s^{\rho-1}}{\left(t^{\rho}-s^{\rho}\right)^{\alpha}} f(a) d s \\
& =\frac{\rho^{\alpha}}{\Gamma(1-\alpha)} \int_{a}^{t}\left(t^{\rho}-s^{\rho}\right)^{-\alpha} f^{\prime}(s) d s .
\end{aligned}
$$

This completes the proof.

Remark 2.12. Let $\rho=1$. We can see that C-K fractional derivative (2.6) is reduced to the classical one, Caputo fractional derivative (2.2). Analogously to the L'Hospital rule, if $f \in C^{1}[a, b]$, then we have

$$
\lim _{\rho \downarrow 0^{+}}{ }^{C} D_{a^{+}}^{\alpha, \rho} f(t)=\frac{1}{\Gamma(1-\alpha)} \int_{a}^{t} \frac{f^{\prime}(s)}{(\ln t-\ln s)^{\alpha}} d s, \quad 0<\alpha<1 .
$$




\section{Maximum and minimum principles}

In this section, we focus our attention to investigate the $\mathrm{C}-\mathrm{K}$ fractional derivatives at extreme points and prove the maximum and minimum principles for fractional $\mathrm{C}-\mathrm{K}$ diffusion operator (1.1). In the rest of the paper, we assume that $a(x, t)>0$ and $c(x, t) \leqslant 0$ for $(x, t) \in(0, l) \times(0, T)$.

The following theorem plays a significant role to obtain the maximum and minimum principles for (1.1), which has been proved by Cao in [5].

Theorem 3.1. If $\mathrm{f} \in \mathrm{C}^{1}[0, \mathrm{~T}]$ obtains the maximum at the point $\mathrm{t}_{0} \in(0, \mathrm{~T})$ and $\alpha \in(0,1]$, then the following inequality holds

$$
{ }^{C} D_{0^{+}}^{\alpha, \rho} f\left(t_{0}\right) \geqslant \frac{\rho^{\alpha} t_{0}^{-\alpha \rho}}{\Gamma(1-\alpha)}\left[f\left(t_{0}\right)-f(0)\right] \geqslant 0 .
$$

Applying Theorem 3.1, we now give the maximum principle for fractional C-K diffusion operator (1.1).

Theorem 3.2. Let $\mathrm{u}:[0, \mathrm{l}] \times[0, \mathrm{~T}] \rightarrow \mathbb{R}$ be such that $\mathrm{u} \in \mathrm{C}^{2,1}\left(\bar{\Omega}_{\mathrm{T}}\right)$ and

$$
\mathrm{P}_{\alpha, \rho}(\mathrm{u}) \leqslant 0, \quad \forall(x, \mathrm{t}) \in \Omega_{\mathrm{T}} .
$$

If $\mathrm{c}(\mathrm{x}, \mathrm{t}) \leqslant 0$ for each $(\mathrm{x}, \mathrm{t}) \in \Omega_{\mathrm{T}}$, then the inequality holds

$$
\max _{(x, t) \in \bar{\Omega}_{T}} u(x, t) \leqslant \max \left\{\max _{(x, t) \in \partial \Omega_{T}} u(x, t), 0\right\},
$$

where $\partial \Omega_{\mathrm{T}}$ is the boundary of rectangle $\Omega_{\mathrm{T}}$.

Proof. If $u$ reaches the maximum at the boundary $\partial \Omega_{\mathrm{T}}$, then it is obvious that (3.1) is satisfied. Otherwise, we can assume that there exists a point $\left(x_{0}, t_{0}\right) \in \Omega_{\mathrm{T}}$ such that

$$
u\left(x_{0}, t_{0}\right)>\max \left\{\max _{(x, t) \in \partial \Omega_{T}} u(x, t), 0\right\}:=M \geqslant 0 .
$$

Then, let us consider the auxiliary function $u^{*}(x, t)$ given by

$$
\mathrm{u}^{*}(x, \mathrm{t}):=\mathrm{u}(\mathrm{x}, \mathrm{t})+\frac{\epsilon}{2} \frac{\mathrm{T}^{\rho}-\mathrm{t}^{\rho}}{\mathrm{T}^{\rho}}, \quad(\mathrm{x}, \mathrm{t}) \in \bar{\Omega}_{\mathrm{T}},
$$

here $\epsilon:=u\left(x_{0}, t_{0}\right)-M>0$.

Therefore, we obtain

$$
\begin{aligned}
{ }^{C} D_{0^{+}}^{\alpha, \rho} u^{*}(x, t) & ={ }^{C} D_{0^{+}}^{\alpha, \rho} u(x, t)+\frac{\epsilon}{2}{ }^{C} D_{0^{+}}^{\alpha, \rho}\left(\frac{T^{\rho}-t^{\rho}}{T^{\rho}}\right) \\
& ={ }^{C} D_{0^{+}}^{\alpha, \rho} u(x, t)-\frac{\epsilon \rho^{\alpha} t^{\rho(1-\alpha)}}{2 T^{\rho} \Gamma(2-\alpha)} .
\end{aligned}
$$

On the other hand, we have

$$
u^{*}(x, t) \leqslant u(x, t)+\frac{\epsilon}{2}, \quad \forall(x, t) \in \bar{\Omega}_{T} .
$$

From the definition of $M$, we can see

$$
u^{*}\left(x_{0}, t_{0}\right)>u\left(x_{0}, t_{0}\right)=M+\epsilon \geqslant \epsilon+u(x, t) \geqslant u^{*}(x, t)+\frac{\epsilon}{2}, \quad \forall(x, t) \in \partial \Omega_{T},
$$


which means that $u^{*}$ cannot reach the maximum on the boundary of $\Omega_{\mathrm{T}}$. We assume $u^{*}$ at $\left(x_{1}, t_{1}\right) \in \Omega_{\mathrm{T}}$ arrives at its maximum value, and we get

$$
u^{*}\left(x_{1}, t_{1}\right) \geqslant u^{*}\left(x_{0}, t_{0}\right)>\epsilon+M \geqslant \epsilon>0 .
$$

According to Theorem 3.1, we have

$$
{ }^{c} D_{0^{+}}^{\alpha, \rho} u^{*}\left(x_{1}, t_{1}\right)={ }^{c} D_{0^{+}}^{\alpha, \rho} u\left(x_{1}, t_{1}\right)-\frac{\epsilon \rho^{\alpha} t_{1}^{\rho(1-\alpha)}}{2 T^{\rho} \Gamma(2-\alpha)} \geqslant 0 .
$$

Furthermore, we can calculate

$$
\begin{aligned}
\mathrm{P}_{\alpha, \rho}\left(\mathrm{u}^{*}\right)\left(\mathrm{x}_{1}, \mathrm{t}_{1}\right) & ={ }^{\mathrm{C}} \mathrm{D}_{0^{+}}^{\alpha, \rho} \mathrm{u}^{*}\left(\mathrm{x}_{1}, \mathrm{t}_{1}\right)-\mathrm{L}\left(\mathrm{u}^{*}\right) \\
& ={ }^{\mathrm{C}} \mathrm{D}_{0^{+}}^{\alpha, \rho} \mathrm{u}^{*}\left(\mathrm{x}_{1}, \mathrm{t}_{1}\right)-\left[\mathrm{a}\left(\mathrm{x}_{1}, \mathrm{t}_{1}\right) \mathrm{u}_{\mathrm{x}}^{*}\left(\mathrm{x}_{1}, \mathrm{t}_{1}\right)+\mathrm{b}\left(\mathrm{x}_{1}, \mathrm{t}_{1}\right) \mathrm{u}_{x}^{*}\left(\mathrm{x}_{1}, \mathrm{t}_{1}\right)+c\left(\mathrm{x}_{1}, \mathrm{t}_{1}\right) \mathrm{u}^{*}\left(\mathrm{x}_{1}, \mathrm{t}_{1}\right)\right],
\end{aligned}
$$

because $u^{*}$ obtains its maximum at $\left(x_{1}, t_{1}\right)$ and $a(x, t)>0, c(x, t) \leqslant 0$ for all $(x, t) \in \bar{\Omega}_{T}$, then we get

$$
u_{x x}^{*}\left(x_{1}, t_{1}\right) \leqslant 0, u_{x}^{*}\left(x_{1}, t_{1}\right)=0, \quad \text { and } u^{*}\left(x_{1}, t_{1}\right) \geqslant \epsilon>0 .
$$

Hence, we derive

$$
P_{\alpha, \rho}\left(u^{*}\right)\left(x_{1}, t_{1}\right) \geqslant-c\left(x_{1}, t_{1}\right) u^{*}\left(x_{1}, t_{1}\right) \geqslant-\epsilon c\left(x_{1}, t_{1}\right)
$$

This means

$$
\begin{aligned}
P_{\alpha, \rho}(u)\left(x_{1}, t_{1}\right) & ={ }^{C} D_{0^{+}}^{\alpha, \rho} u\left(x_{1}, t_{1}\right)-L(u) \\
& ={ }^{C} D_{0^{+}}^{\alpha, \rho} u^{*}\left(x_{1}, t_{1}\right)-L\left(u^{*}\right)+\frac{\epsilon \rho^{\alpha} t_{1}^{\rho(1-\alpha)}}{2 T^{\rho} \Gamma(2-\alpha)}+\frac{\epsilon\left(T^{\rho}-t_{1}^{\rho}\right)}{2 T^{\rho}} c\left(x_{1}, t_{1}\right) \\
& \geqslant \frac{\epsilon \rho^{\alpha} t_{1}^{\rho(1-\alpha)}}{2 T^{\rho} \Gamma(2-\alpha)}+\frac{\epsilon\left(T^{\rho}-t_{1}^{\rho}\right)}{2 T^{\rho}} c\left(x_{1}, t_{1}\right)-\epsilon c\left(x_{1}, t_{1}\right), \\
& \geqslant \frac{\epsilon \rho^{\alpha} t_{1}^{\rho(1-\alpha)}}{2 T^{\rho} \Gamma(2-\alpha)}-\epsilon c\left(x_{1}, t_{1}\right)\left(1-\frac{T^{\rho}-t_{1}^{\rho}}{T^{\rho}}\right) \\
& >0
\end{aligned}
$$

which contradicts with the fact, $P_{\alpha, \rho}(u)(x, t) \leqslant 0$ for all $(x, t) \in \Omega_{\top}$. Consequently, we conclude that (3.1) holds.

Similarly, we can present the minimum principle for fractional C-K diffusion operator (1.1).

Theorem 3.3. Let $\mathrm{u}:[0, \mathrm{l}] \times[0, \mathrm{~T}] \rightarrow \mathbb{R}$ be such that $\mathrm{u} \in \mathrm{C}^{2,1}\left(\bar{\Omega}_{\mathrm{T}}\right)$ and

$$
\mathrm{P}_{\alpha, \rho}(\mathrm{u}) \geqslant 0, \quad \forall(x, t) \in \Omega_{\mathrm{T}} .
$$

If $\mathrm{c}(\mathrm{x}, \mathrm{t}) \leqslant 0$ for each $(\mathrm{x}, \mathrm{t}) \in \Omega_{\mathrm{T}}$, then the inequality holds

$$
\min _{(x, t) \in \bar{\Omega}_{T}} u(x, t) \geqslant \min \left\{\min _{(x, t) \in \partial \Omega_{T}} u(x, t), 0\right\} .
$$

According to Theorem. 3.2 and Theorem. 3.3, we have results in the case of $P_{\alpha, \rho}(u)=0$.

Theorem 3.4. Let $\mathrm{u}:[0, \mathrm{l}] \times[0, \mathrm{~T}] \rightarrow \mathbb{R}$ be such that $\mathrm{u} \in \mathrm{C}^{2,1}\left(\bar{\Omega}_{\mathrm{T}}\right)$ and

$$
\mathrm{P}_{\alpha, \rho}(\mathrm{u})=0, \quad \forall(x, \mathrm{t}) \in \Omega_{\mathrm{T}} .
$$

If $\mathrm{c}(\mathrm{x}, \mathrm{t}) \leqslant 0$ for each $(\mathrm{x}, \mathrm{t}) \in \Omega_{\mathrm{T}}$, and the function reaches the maximum and minimum at a few points that are part of $\Omega_{\mathrm{T}}$, subsequently the function $\mathrm{u}$ is a constant, that is $\mathrm{u}(\mathrm{x}, \mathrm{t})=0,(\mathrm{x}, \mathrm{t}) \in \bar{\Omega}_{\mathrm{T}}$. 


\section{Applications of the maximum principles}

In this section, we apply the main results in Section 3 to prove the properties of solution for onedimensional time-fractional C-K diffusion equation.

First, we focus our attention to investigate the one-dimensional time-fractional C-K linear diffusion equation as follows

$$
{ }^{c} D_{0^{+}}^{\alpha, \rho} u(x, t)-L(u)=f(x, t), \quad(x, t) \in \Omega_{T},
$$

and it satisfies the initial-boundary conditions

$$
\left\{\begin{array}{l}
u(0, t)=g_{1}(t), \quad t \in[0, T], \\
u(l, t)=g_{2}(t), \quad t \in[0, T],
\end{array}\right.
$$

and

$$
u(x, 0)=\Phi(x), x \in[0, l] .
$$

From the maximum and minimum principles, see Theorems 3.2 and 3.3, we can get the results as follows.

Theorem 4.1. Assume $\mathrm{f}(\mathrm{x}, \mathrm{t}) \leqslant 0$ for all $(\mathrm{x}, \mathrm{t}) \in \Omega_{\mathrm{T}}, \Phi(\mathrm{x}) \leqslant 0$ for all $\mathrm{x} \in[0, \mathrm{l}] ; \mathrm{g}_{1}(\mathrm{t}) \leqslant 0$ and $\mathrm{g}_{2}(\mathrm{t}) \leqslant 0$ for all $\mathrm{t} \in[0, \mathrm{~T}], \mathrm{c}(\mathrm{x}, \mathrm{t}) \leqslant 0$ for all $(\mathrm{x}, \mathrm{t}) \in \Omega_{\mathrm{T}}$. If $\mathrm{u} \in \mathrm{C}^{2,1}\left(\bar{\Omega}_{\mathrm{T}}\right)$ is a solution of problem (4.1) with initial condition (4.3) and boundary conditions (4.2), then

$$
u(x, t) \leqslant 0, \quad(x, t) \in \bar{\Omega}_{\mathrm{T}} .
$$

Hence, the problem (4.1), (4.2), (4.3) does not possess any positive solutions in $\mathrm{C}^{2,1}\left(\bar{\Omega}_{\mathrm{T}}\right)$.

Proof. By using Theorem 3.2, we easily get this theorem.

Theorem 4.2. Assume $\mathrm{f}(\mathrm{x}, \mathrm{t}) \geqslant 0$ for all $(\mathrm{x}, \mathrm{t}) \in \Omega_{\mathrm{T}}, \Phi(\mathrm{x}) \geqslant 0$ for all $\mathrm{x} \in[0, \mathrm{l}], \mathrm{g}_{1}(\mathrm{t}) \geqslant 0$ and $\mathrm{g}_{2}(\mathrm{t}) \geqslant 0$ for all $\mathrm{t} \in[0, \mathrm{~T}], \mathrm{c}(\mathrm{x}, \mathrm{t}) \leqslant 0$ for every $(\mathrm{x}, \mathrm{t}) \in \Omega_{\mathrm{T}}$. If $\mathrm{u} \in \mathrm{C}^{2,1}\left(\bar{\Omega}_{\mathrm{T}}\right)$ is a solution of problem (4.1) with initial condition (4.3) and boundary condition (4.2), then

$$
u(x, t) \geqslant 0, \quad(x, t) \in \bar{\Omega}_{\mathrm{T}},
$$

thus we obtain the problem (4.1), (4.2), (4.3) does not possess any negative solutions in $\mathrm{C}^{2,1}\left(\bar{\Omega}_{\mathrm{T}}\right)$.

Proof. From Theorem 3.2, we can directly prove this theorem.

Remark 4.3. In fact, if $u \in \mathrm{C}^{2,1}\left(\bar{\Omega}_{\mathrm{T}}\right)$ is a solution of the problem (4.1) satisfying initial conditions (4.3) and boundary conditions (4.2) with $f(t, x)=0$ for all $(t, x) \in \bar{\Omega}_{T}$, then the C-K linear diffusion equation (4.1), (4.2), (4.3) has only zero solution in $\mathrm{C}^{2,1}\left(\bar{\Omega}_{\mathrm{T}}\right)$.

Next, we focus our attention on the $\mathrm{C}-\mathrm{K}$ nonlinear diffusion equation as follows

$$
{ }^{C} D_{0^{+}}^{\alpha, \rho} u(x, t)-L(u)=F(x, t, u), \quad(x, t) \in \Omega_{\mathrm{T}} .
$$

Theorem 4.4. If $\mathrm{F}:[0, \mathrm{l}] \times[0, \mathrm{~T}] \times \mathbb{R} \rightarrow \mathbb{R}$ is such that $\partial_{\mathfrak{u}} \mathrm{F}=\partial_{\mathfrak{u}} \mathrm{F}(\mathrm{x}, \mathrm{t}, \mathrm{u})$ exists and satisfies $\partial_{\mathfrak{u}} \mathrm{F}(\mathrm{x}, \mathrm{t}, \mathrm{u})+$ $c(x, t) \leqslant 0$ for all $(x, t, u) \in \Omega_{T} \times \mathbb{R}$, then time-fractional $C-K$ nonlinear diffusion equation (4.4) with the initial condition (4.3) and boundary conditions (4.2) owns at most one solution in the function space $C^{2,1}\left(\bar{\Omega}_{\mathrm{T}}\right)$.

Proof. Arguing by contradiction, we assume that the nonlinear diffusion equation (4.4) has two distinct solutions $u_{1}$ and $u_{2}$ with the initial condition (4.3) and boundary conditions (4.2), which pertain to the function space $C^{2,1}\left(\bar{\Omega}_{T}\right)$. Denote $u(x, t)=u_{1}(x, t)-u_{2}(x, t),(x, t) \in \bar{\Omega}_{T}$. We easily get

$$
{ }^{C} D_{0^{+}}^{\alpha, \rho} u(x, t)=a(x, t) u_{x x}(x, t)+b(x, t) u_{x}(x, t)+c(x, t) u(x, t)+F\left(t, x, u_{1}\right)-F\left(t, x, u_{2}\right) .
$$

In addition, the function $u$ satisfies the homogeneous initial-boundary conditions,

$$
u(x, t)=0, \quad(x, t) \in \partial \Omega_{T} .
$$


Because the partial derivative $\partial_{\mathfrak{u}} F=\partial_{\mathfrak{u}} F(x, t, u)$ exists, we use the mean value theorem that

$$
F\left(t, x, u_{1}\right)-F\left(t, x, u_{2}\right)=\frac{\partial F}{\partial u}\left(u^{*}\right)\left(u_{1}(x, t)-u_{2}(x, t)\right), \quad \forall(x, t) \in \Omega,
$$

where $u^{*}=\lambda u_{1}+(1-\lambda) u_{2}$, for some $\lambda \in[0,1]$.

Therefore, we have

$$
\left\{\begin{array}{l}
{ }^{c} D_{0^{+}}^{\alpha, \rho} u(x, t)=a(x, t) u_{x x}(x, t)+b(x, t) u_{x}(x, t)+h(x, t) u(x, t), \quad(x, t) \in \Omega_{T}, \\
u(x, t)=0, \quad(x, t) \in \partial \Omega_{T},
\end{array}\right.
$$

where $h(x, t):=\frac{\partial F}{\partial u}\left(u^{*}\right)+c(x, t)$ for all $(x, t) \in \Omega_{\mathrm{T}}$.

By using Theorems 4.1 and 4.2 , one has

$$
u(x, t) \leqslant 0 \text { and } u(x, t) \geqslant 0, \quad(x, t) \in \bar{\Omega}_{T} .
$$

This means $u(x, t)=0$ for all $(x, t) \in \bar{\Omega}_{T}$, i.e., $u_{1}=u_{2}$. Consequently, we prove that time-fractional C-K nonlinear diffusion equation (4.4) with initial condition (4.3) and boundary condition (4.2) owns no more than one solution within the function space $C^{2,1}\left(\bar{\Omega}_{\mathrm{T}}\right)$.

Remark 4.5. The condition $\partial_{\mathfrak{u}} \mathrm{F}(\mathrm{x}, \mathrm{t}, \mathrm{u})+\mathrm{c}(\mathrm{x}, \mathrm{t}) \leqslant 0$ plays a significant role. In fact, if $\mathrm{F}$ is a nonincreasing function in regard to the third variable, then $\partial_{\mathfrak{u}} F(x, t, u)+c(x, t) \leqslant 0$ is obviously satisfied.

Corollary 4.6. If $\mathrm{c}(\mathrm{x}, \mathrm{t}) \leqslant 0,(\mathrm{x}, \mathrm{t}) \in \Omega_{\mathrm{T}}$, then time-fractional linear $C-K$ diffusion equation (4.1) with boundary condition (4.2) and initial condition (4.3) has no more than one solution within the function space $\mathrm{C}^{2,1}\left(\bar{\Omega}_{\mathrm{T}}\right)$.

In the end, we present some applications of the maximum principles about stability results for solution of the linear initial-boundary-valued (4.1), (4.2), (4.3).

Theorem 4.7. Let $\mathrm{u}_{1}(\mathrm{x}, \mathrm{t})$ and $\mathrm{u}_{2}(\mathrm{x}, \mathrm{t}) \in \mathrm{C}^{2,1}\left(\bar{\Omega}_{\mathrm{T}}\right)$ be two solutions of the time-fractional linear $C-K$ diffusion equation (4.1) that gratify the same boundary conditions (4.2), and initial conditions $u_{1}(x, 0)=\Phi_{1}(x)$ and $\mathrm{u}_{2}(x, 0)=\Phi_{2}(x), x \in[0, l]$, respectively. If $\mathrm{c}(x, t) \leqslant 0$ for all $(x, t) \in \Omega_{T}$, then the inequality is satisfied

$$
\max _{(x, t) \in \bar{\Omega}_{T}}\left|u_{1}(x, t)-u_{2}(x, t)\right| \leqslant \max _{x \in[0, l]}\left|\Phi_{1}(x)-\Phi_{2}(x)\right| .
$$

Proof. Since $u_{1}, u_{2} \in C^{2,1}\left(\bar{\Omega}_{T}\right)$ are two solutions of the linear diffusion equation (4.1), then we have

$$
\begin{cases}{ }^{c} D_{0^{+}}^{\alpha, \rho} u_{i}=L(u)+f(x, t), & (x, t) \in \Omega_{T}, \\ u_{i}(0, t)=g_{1}(t), & t \in[0, T], \\ u_{i}(l, t)=g_{2}(t), & t \in[0, T], \\ u_{i}(x, 0)=\Phi_{i}(x), & x \in[0, l],\end{cases}
$$

where $i=1,2$.

Let $u=u_{1}-u_{2}$. One yields

$$
\begin{cases}{ }^{c} D_{0^{+}}^{\alpha, \rho} u=L(u), & (x, t) \in \Omega_{\mathrm{T}}, \\ u(0, t)=u(l, t)=0, & t \in[0, T], \\ u(x, 0)=\Phi_{1}(x)-\Phi_{2}(x), & x \in[0, l] .\end{cases}
$$

Using the maximum and minimum principles, i.e., Theorems 3.2 and 3.3, we conclude

$$
\max _{(x, t) \in \bar{\Omega}_{T}} u(x, t) \leqslant \max \left\{\max _{x \in[0, l]}\left(\Phi_{1}(x)-\Phi_{2}(x)\right), 0\right\},
$$


and

$$
\min _{(x, t) \in \bar{\Omega}_{T}} u(x, t) \geqslant \min \left\{\min _{x \in[0, l]}\left(\Phi_{1}(x)-\Phi_{2}(x)\right), 0\right\}
$$

respectively.

Combining (4.5) and (4.6), we get

$$
\max _{(x, t) \in \bar{\Omega}_{T}}|\mathfrak{u}(x, t)|=\max _{(x, t) \in \bar{\Omega}_{T}}\left|u_{1}(x, t)-u_{2}(x, t)\right| \leqslant \max _{(x, t) \bar{\Omega}_{T}}\left|\Phi_{1}(x)-\Phi_{2}(x)\right| .
$$

This completes the proof.

Theorem 4.8. Let $\mathrm{u}_{1}(x, \mathrm{t})$ and $\mathrm{u}_{2}(x, \mathrm{t}) \in \mathrm{C}^{2,1}\left(\bar{\Omega}_{\mathrm{T}}\right)$ be two solutions of the time-fractional $\mathrm{C}-\mathrm{K}$ linear diffusion equation (4.1) that satisfy the same initial condition (4.3) and boundary conditions

$$
\left\{\begin{array}{l}
\mathrm{u}_{1}(0, \mathrm{t})=\bar{g}_{1}(\mathrm{t}), \quad \mathrm{t} \in[0, \mathrm{~T}], \\
\mathrm{u}_{1}(\mathrm{l}, \mathrm{t})=\bar{g}_{2}(\mathrm{t}),
\end{array}\right.
$$

and

$$
\left\{\begin{array}{l}
\mathrm{u}_{2}(0, \mathrm{t})=\widehat{\mathrm{g}}_{1}(\mathrm{t}), \\
\mathrm{u}_{2}(\mathrm{l}, \mathrm{t})=\widehat{\mathrm{g}}_{2}(\mathrm{t}),
\end{array} \quad \mathrm{t} \in[0, \mathrm{~T}],\right.
$$

respectively. If $\mathrm{c}(\mathrm{x}, \mathrm{t}) \leqslant 0$ for all $(\mathrm{x}, \mathrm{t}) \in \Omega_{\mathrm{T}}$, then the following inequality holds

$$
\max _{(x, t) \in \bar{\Omega}_{T}}\left|u_{1}(x, t)-u_{2}(x, t)\right| \leqslant \max \left\{\max _{t \in[0, T]}\left|\bar{g}_{1}(t)-\widehat{g}_{1}(t)\right|, \max _{t \in[0, T]}\left|\bar{g}_{2}(t)-\widehat{g}_{2}(t)\right|\right\} .
$$

Proof. By the same arguments as that in Theorem 4.7 , let $u=u_{1}-u_{2}$, then

$$
\begin{cases}{ }^{C} D_{0^{+}}^{\alpha, \rho} u(x, t)=L(u), & (x, t) \in \Omega_{T}, \\ u(0, t)=\bar{g}_{1}(t)-\widehat{g}_{1}(t), & t \in[0, T], \\ u(L, t)=\bar{g}_{2}(t)-\widehat{g}_{2}(t), & t \in[0, T], \\ u(x, 0)=0, \quad x \in[0, l] . & \end{cases}
$$

Applying the maximum and minimum principles again (see, Theorem 3.2 and Theorem 3.3), we can obtain

$$
\max _{(x, t) \in \bar{\Omega}_{T}}\left(u_{1}(x, t)-u_{2}(x, t)\right) \leqslant \max \left\{\max _{t \in[0, T]}\left\{\bar{g}_{1}(t)-\widehat{g}_{1}(t)\right\}, \max _{t \in[0, T]}\left\{\bar{g}_{2}(t)-\widehat{g}_{2}(t)\right\}, 0\right\},
$$

and

$$
\min _{(x, t) \in \bar{\Omega}_{T}}\left(u_{1}(x, t)-u_{2}(x, t)\right) \geqslant \min \left\{\min _{t \in[0, T]}\left\{\bar{g}_{1}(x)-\widehat{g}_{1}(t)\right\}, \min _{t \in[0, T]}\left\{\bar{g}_{2}(t)-\widehat{g}_{2}(t)\right\}, 0\right\},
$$

respectively.

As a result, we obtain

$$
\max _{(x, t) \in \bar{\Omega}_{T}}\left|u_{1}(x, t)-u_{2}(x, t)\right| \leqslant \max \left\{\max _{t \in[0, T]}\left|\bar{g}_{1}(x)-\widehat{g}_{1}(t)\right|, \max _{t \in[0, T]}\left|\bar{g}_{2}(x)-\widehat{g}_{2}(t)\right|\right\} .
$$

This completes the proof.

\section{Conclusions}

In this study, we mainly develop the maximum principles for fractional partial differential equations. We first introduce the C-K derivatives and give the relationship between $\mathrm{C}-\mathrm{K}$ fractional operators, Caputo fractional operators and Caputo-Hadamard fractional operators. Then we establish the maximum principles for time-fractional C-K diffusion operators. Finally, these principles are employed to derive the uniqueness of solutions of the time-fractional $\mathrm{C}-\mathrm{K}$ diffusion equations and continuous dependence of solutions on initial-boundary value conditions. 


\section{Acknowledgment}

This work was financially supported by the National Natural Science Foundation of China (Grant No. 11301257) and the Innovative Team Program of the Neijiang Normal University (Grant No. 13TD02).

\section{References}

[1] M. Al-Refai, Y. Luchko, Maximum principle for the fractional diffusion equations with the Riemann-Liouville fractional derivative and its applications, Fract. Calc. Appl. Anal., 17 (2014), 483-498. 1

[2] M. Al-Refai, Y. Luchko, Maximum principle for the multi-term time-fractional diffusion equations with the RiemannLiouville fractional derivatives, Appl. Math. Comput., 257 (2015), 40-51. 1, 1

[3] A. H. Bhrawy, M. A. Zaky, A method based on the Jacobi tau approximation for solving multi-term time-space fractional partial differential equations, J. Comput. Phys., 281 (2015), 876-895. 1

[4] P. L. Butzer, A. A. Kilbas, J. J. Trujillo, Fractional calculus in the Mellin setting and Hadamard-type fractional integrals, J. Math. Anal. Appl., 269 (2002), 1-27. 1

[5] L. Cao, Maximum principle theorem for Caputo-Katugampola fractional operators, J. Comput. Complex. Appl., 3 (2017), 68-71. 3

[6] A. R. Carella, C. A. Dorao, Least-squares spectral method for the solution of a fractional advection-dispersion equation, J. Comput. Phys., 232 (2013), 33-45. 1

[7] R. Gorenflo, Y. Luchko, M. Yamamoto, Time-fractional diffusion equation in the fractional Sobolev spaces, Fract. Calc. Appl. Anal., 18 (2015), 799-820. 1

[8] U. N. Katugampola, New approach to a generalized fractional integral, Appl. Math. Comput., 218 (2011), 860-865. 2

[9] U. N. Katugampola, A new approach to generalized fractional derivatives, Bull. Math. Anal. Appl., 6 (2014), 1-15. 2

[10] A. A. Kilbas, H. M. Srivastava, J. J. Trujillo, Theory and applications of fractional differential equations, North-Holland Mathematics Studies, Elsevier Science B.V., Amsterdam, (2006). 2, 2

[11] Z.-H. Liu, S.-D. Zeng, Y.-R. Bai, Maximum principles for multi-term space-time variable-order fractional diffusion equations and their applications, Fract. Calc. Appl. Anal., 19 (2016), 188-211. 1

[12] Y. Luchko, Maximum principle for the generalized time-fractional diffusion equation, J. Math. Anal. Appl., 351 (2009), 218-223. 1, 1

[13] Y. Luchko, Initial-boundary-value problems for the one-dimensional time-fractional diffusion equation, Fract. Calc. Appl. Anal., 15 (2012), 141-160. 1

[14] Y. Luchko, W. Rundell, M. Yamamoto, L.-H. Zou, Uniqueness and reconstruction of an unknown semilinear term in a time-fractional reaction-diffusion equation, Inverse Problems, 29 (2013), 16 pages. 1

[15] F. Mainardi, Fractional calculus and waves in linear viscoelasticity, An introduction to mathematical models. Imperial College Press, London, (2010). 1

[16] S. Momani, Z. Odibat, Analytical approach to linear fractional partial differential equations arising in fluid mechanics, Phys. Lett. A, 355 (2006), 271-279. 1

[17] I. Podlubny, Fractional differential equations, An introduction to fractional derivatives, fractional differential equations, to methods of their solution and some of their applications, Mathematics in Science and Engineering, Academic Press, Inc., San Diego, CA, (1999). 2, 2

[18] Y. Povstenko, Non-axisymmetric solutions to time-fractional diffusion-wave equation in an infinite cylinder, Fract. Calc. Appl. Anal., 14 (2011), 418-435. 1

[19] S. G. Samko, A. A. Kilbas, O. I. Marichev, Fractional integrals and derivatives, Theory and applications, Edited and with a foreword by S. M. Nikolskiü, Translated from the 1987 Russian original, Revised by the authors, Gordon and Breach Science Publishers, Yverdon, (1993). 2, 2

[20] A. Suzuki, Y. Niibori, S. Fomin, V. Chugunov, T. Hashida, Prediction of reinejction effects in fault-related subsidiary structures by using fractional derivative-based mathematical models for sustainable design of geothermal reservoirs, Geothermics, 57 (2015), 196-204. 1, 2, 2

[21] G.-C. Wu, D. Baleanu, Z.-G. Deng, S.-D. Zeng, Lattice fractional diffusion equation in terms of a Riesz-Caputo difference, Phys. A, 438 (2015), 335-339. 1

[22] G.-C. Wu, D. Baleanu, S.-D. Zeng, Discrete chaos in fractional sine and standard maps, Phys. Lett. A, 378 (2014), 484-487. 1

[23] X.-J. Yang, D. Baleanu, M. C. Baleanu, Observing diffusion problems defined on Cantor sets in different co-ordinate systems, Therm. Sci., 19 (2015), S151-S156. 1

[24] X.-J. Yang, D. Baleanu, Y. Khan, S. T. Mohyud-Din, Local fractional variational iteration method for diffusion and wave equations on Cantor sets, Romanian J. Phys., 59 (2014), 36-48.

[25] X.-J. Yang, D. Baleanu, H. M. Srivastava, Local fractional integral transforms and their applications, Elsevier/Academic Press, Amsterdam, (2015).

[26] X.-J. Yang, D. Baleanu, H. M. Srivastava, Local fractional similarity solution for the diffusion equation defined on Cantor sets, Appl. Math. Lett., 47 (2015), 54-60. 
[27] X.-J. Yang, D. Baleanu, W.-P. Zhong, Approximate solutions for diffusion equations on Cantor space-time, Proc. Rom. Acad. Ser. A Math. Phys. Tech. Sci. Inf. Sci., 14 (2013), 127-133.

[28] X.-J. Yang, Z.-Z. Zhang, J. A. T. Machado, D. Baleanu, On local fractional operators view of computational complexity: diffusion and relaxation defined on cantor sets, Therm. Sci., 20 (2016), S755-S767. 1

[29] H. Ye, F. Liu, V. Anh, I. Turner, Maximum principle and numerical method for the multi-term time-space Riesz-Caputo fractional differential equations, Appl. Math. Comput., 227 (2014), 531-540. 1, 1

[30] P. Zhuang, F. Liu, V. Anh, I. Turner, Numerical methods for the variable-order fractional advection-diffusion equation with a nonlinear source term, SIAM J. Numer. Anal., 47 (2009), 1760-1781. 1 